\title{
Research on Consumers' Online Purchase Decision based on Psychological Distance
}

\author{
Tiantian Tang ${ }^{1,}$, Pei $\mathrm{Hu}^{1, \mathrm{~b}}$ \\ ${ }^{1}$ School of Economics and Management, Southwest Jiaotong University, Chengdu, 610031 \\ ${ }^{a}$ email, ${ }^{b}$ email
}

Keywords: Consumer Online Purchase Decision, Psychological Distance

\begin{abstract}
Online consumers make online shopping decisions that are a complex process that can be influenced by a variety of factors, which factors affect and how they affect purchasing decisions. For example, online consumers to browse the site on the product information, online reviews and other information, access to different types of information perception (abstract, overall, summary or specific, local, details) of its decision-making is the same? The geographical location of the network business or the length of logistics and distribution of goods will also have an impact on decision-making? Their online shopping and online shopping for others to provide advice compared to the decision made by consumers are the same? And, when consumers face purchasing decisions, the above factors are likely to have an impact on them at the same time. For example, in the face of different characteristics of goods information, when in the near future and far future, for their own decision-making and decision-making for others to provide recommendations are the same? How to combine these factors to influence the purchasing decision of online consumers is very few. At present, the research on the influence of psychological distance and explanation level on consumer purchasing decision has become an urgent requirement for the development of e-commerce.
\end{abstract}

\section{Introduction}

In the rapid development of information technology today, e-commerce and the traditional trade with different cross-distance trade model, changing the traditional market competition, bringing new opportunities and challenges. With the rapid development of network information technology and the rapid popularization of online shopping, online shopping will gradually become one of the main ways of shopping, e-commerce has become an important model of commodity trading, the major domestic shopping site frequent promotions also inspired Consumer demand for new purchases, driven by the steady growth of the scale of online shopping users, the domestic online shopping market showed a rapid expansion of the trend. According to the China Electronic Commerce Research Center released the "China e-commerce market data monitoring report" shows that as of last year, the national e-commerce transactions amounted to one trillion yuan, China's online shopping users reached 100 million scale, the network retail market Billion, the annual growth in the first half of the year, China's online retail market transactions accounted for the total retail sales of social consumer goods. Traditional businessmen gradually realized that the online market is an important area to expand market share, the network sales as a new marketing methods, have set foot in the network for product sales, with the network to establish a virtual shop to engage in commercial activities, resulting in online supply market Expansion, online retailers have also entered the competition between the white-hot stage, competition between various types of commercial sites intensified. The convenience of the network and the borderlessness of the Internet on the one hand to get rid of the time and space fish, on the other hand provides a wealth of information resources, so the transfer costs of network consumers is very low, according to China's Internet hot report finishing found: network consumers In the shopping, some consumers will be in a number of sites between the goods comparison, in comparison to the online mall prices, distribution, payment, service and other factors before making a purchase decision. Therefore, for the network business, in order to profit in the network sales and competitive advantage, to accurately grasp the psychological changes in consumer online shopping and features is particularly 
important.

\section{Analysis on the Influence Mechanism of Psychological Distance on Online Shopping Decision}

The information provided by the merchant is a kind of external stimulus information for the consumers who are making the purchase decision. This kind of information has a certain effect on the consumer's judgment and decision-making, and how the consumer is closely related to the psychological representation, The level of interpretation of the information may be influenced by the psychological distance (intrinsic mental state) of the consumer's decision. This matching consistency will lead to different concerns about the information provided by the consumer, resulting in different preferences and influencing their decision making. When the information provided by the business and the psychological distance of the consumer match the state, the consumer will be aware of the information when dealing with a higher fluency, thereby enhancing the effectiveness of information and consumer evaluation of the product. In other words, the consumer perceives that the information that matches the expectation will increase the satisfaction level, and the likelihood of its purchase decision is improved with the consistent match of the information interpretation level and the psychological distance. On the contrary, if the psychological distance of the consumer and the information provided by the business is inconsistent when the consumer receives the information processed processing, will produce a feeling of poor fluency, the satisfaction of the information obtained, may be in the psychological distance More distant, so consumers of the product's positive evaluation and purchase desire may be reduced. To sum up, based on the theory of cognitive consistency matching, when the psychological distance between consumers and the interpretation of the level of matching, consumers tend to use the same type of information as a decision-making benchmark, so that the probability of purchase will be relatively large probability. That is, when the consumer online shopping decision-making psychological distance and the level of information between the level of matching affect the consumer's purchase decision. Specifically, the information type and the psychological distance of the high degree of match is reflected in: when the consumer and the network business goods psychological distance, consumers tend to enable low-level interpretation of the psychological representation model, on the contrary, when the psychological distance In the distant days, consumers tend to enable a high level of interpretation of the psychological representation model.

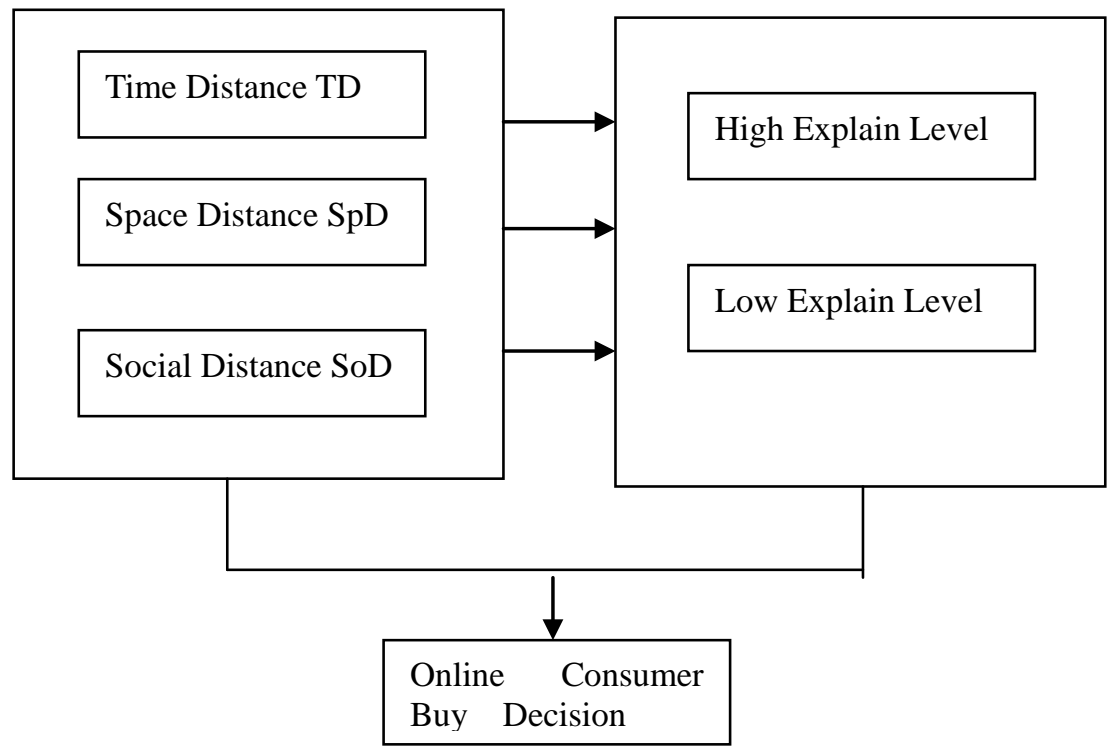

Figure 1 Single-dimensional psychological distance on the impact of online shopping decision-making framework

In summary, when the psychological distance of the consumer is different from the network of goods at the same time the current information, they tend to integrate through the comprehensive assessment, due to synergies, different aspects of attention and weight will be enhanced, the consumer Greater impact. According to the idea of coherence theory, the psychological distance and 
the level of interpretation or match will promote each other, and inconsistent negative effect will weaken the positive impact, that psychological distance and sales of goods on the level of perception is not independent. Not linear superposition, but the existence of interaction. In short, the psychological distance and the level of interpretation of commodity information or consistency will affect the consumer's purchase decision.

\section{Research on the Influence of Psychological Distance on Online Shopping Decision}

Online consumers in the actual online shopping, usually do not only contain a psychological distance of a dimension, often related to the psychological distance of multiple dimensions. Such as consumers in the shopping site browsing, fancy two businesses (a distance and the other closer) of the product (a major or abstract features better and another secondary or specific characteristics of better), tomorrow Buy or other people in a month to buy their own to provide advice, the decision will make the same? In this scenario, the three dimensions of the psychological distance - the time distance, the social distance and the spatial distance, and the level of interpretation of the commodity are at the same time. We speculate that when the distance between these distance dimensions forms a new psychological distance level that matches the level of interpretation of the network commodity, it may have some degree of impact on the decision of the online consumer. To verify the experimental assumptions in Chapter 4, we have designed three experiments in this chapter. By simulating the environment of online consumer online shopping, the experimental method of situational simulation is used to select different experimental stimuli and scenario design. From the perspective of three dimensions of psychological distance, the psychological distance is analyzed to influence the purchasing decision of online consumers The In order to increase the external validity, the second group and the third group experiment were selected by digital products (mobile phone), online English, and so on. In the second group, the second group and the third group were selected as the experimental materials, and the subjects were affected by the interaction of time distance, space distance and social distance. Oral training as an experimental material, while manipulating the subjects of the time distance, space distance and social distance and the level of interpretation of goods to study the psychological distance on the impact of online consumer purchasing decisions.

In the context of the study in this paper, the three dimensions of time distance, spatial distance and social distance are involved in the process of online shopping, and play a role in the process of online shopping. This chapter validates the influence of these three dimensions on the psychological distance and the level of commodity interpretation at the same time on the decision of online consumers.In order to enhance the external validity of the research, we use a variety of network product categories.This study, through three scenario simulation experiments, Experience the relationship between the product, the search product, the point of view of the trust product, the distance between the study distance, the spatial distance and the social distance dimension, and the interaction between them and the interpretation level, and discusses the influence of the psychological distance on the online consumer purchase decision The results show that when the online consumers make decisions, they will be affected by the interaction of the psychological distance between the dimensions and their explanatory level.The three psychological distance dimensions interact to form a new psychological distance, which is perceived by the consumers Consumers are most likely to make the same level of information on high and low levels of online goods Specifically, if and only if the time distance, the spatial distance, the social distance, the three distance dimensions are at the same time close to the consumer's low interpretation level perception, or only when the three distance dimensions are at the same time Consumers are most likely to have a purchasing decision when the consumer's high level of explanation matches, and from the three experimental results in this chapter, the product category does not have a significant impact.

\section{Conclusion}

When the online consumers are online shopping, the psychological distance between the two 
dimensions is related to the psychological distance of the two dimensions, and the level of the interpretation of the commodity has an interactive effect on the online shopping decision of the consumer. The result shows that the two satisfy the matching degree to a certain extent. Time and social distance are both close, consumers are more concerned about the low level of explanation, these attributes will have a more positive influence, online consumers are more likely to rely on these simple external, local information clues to select and judge , The possibility of making decisions is greater; when a distance is far or two distance dimensions are far, consumers are more sensitive to the high level of interpretation, that the main features of the network commodity that is highly attractive level of consumption, online consumption Will be based on the main overall characteristics of the network of goods thinking, in this case will be more likely to produce the purchase intention. However, the experimental study in this paper finds that the influence of time distance and spatial distance, social distance and spatial distance on decision-making does not accord with the above-mentioned laws. When the time distance and the spatial distance, the social distance and the spatial distance are only coincident with the low level of interpretation of the commodity, or the above two dimensions are at the same time and are consistent with the high level of perception of the commodity Making it more likely to make a purchase decision.

\section{Acknowledgements}

Major Projects of National Natural Science Foundation of China (Grant No. 71090402): Research on the Behaviors of the Participants of Service and Service Strategy under the Network Environment. National Natural Science Foundation of China (Grant No. 71002063): Research on the Mechanism and Boundary Conditions of Sales Promotion's Influence on Consumers' Purchasing Decisions and Brand Equity.

\section{References}

[1] Du Weiyu, Jiang Yunxia. The effect of consumer's explanation level on its preference [J] .Journal of Psychology. 2014 (01)

[2] Su glaze, Huang Jinsong. The effect of reverse marketing: based on the perspective of CLT theory [J]. Management of the world. 2013 (11)

[3] He Yun. Time distance and interpretation of the impact of the evaluation of sponsorship [J]. Management Review. 2013 (10)

[4] Huang He-ting, zhao Dong-mei. Study on the Psychological Distance of Online Consumers and Its Measuring Methods - Based on the Theory of Interpretation Level [J] .Journal of Economic Research. 2013 (14)

[5] Hua Shengxu, Lu Houchao. The relationship between psychological distance and construction level [J] .Journal of Psychology. 2012 (06) 